EPJ Web of Conferences 41, 10022 (2013)

DOI: $10.1051 /$ epjconf/20134110022

(C) Owned by the authors, published by EDP Sciences, 2013

\title{
Measuring the suppression of ultrashort pulses into Airy-Bessel light bullets with almost single-cycle temporal resolution
}

\author{
P. Piksarv, H. Valtna-Lukner, A. Valdmann, M. Lõhmus, R. Matt, and P. Saari \\ Institute of Physics, University of Tartu, Riia 142, Tartu, 51014 Estonia
}

\begin{abstract}
We have achieved $\sim 3$ fs and $\sim 3 \mu \mathrm{m}$ resolutions for full spatio-temporal characterization of impulse responses of optical systems using a white-light spectral interferometry set-up and demonstrate how a circular diffraction grating temporally focuses an Airy-Bessel wave-packet.
\end{abstract}

\section{Introduction}

Recent advanced ultrashort pulse characterization techniques have enabled the measurement of the complex light-field simultaneously in both spatial and temporal domain. In addition to careful dispersion compensation and pulse compression techniques, high-resolution spatio-temporal detection of ultrashort laser pulses is required for obtaining pulses with a spectrum spanning over the visible region and lasting only about one optical cycle and focused down to the diffraction limit. Such a high spatio-temporal localization of laser pulses is much desired for numerous applications in non-linear optics, imaging, lithography, communications, etc.

For example, one such measurement method is SEA TADPOLE. It has proved as a useful tool for measuring focusing and diffraction properties of ultrashort pulses as well as various "nondiffracting" pulses [1-5]. Here, we propose a version of spatio-spectral interferometry technique employing supercontinuum laser source and photonic crystal fibres for full spatio-temporal measurement of impulse responses of optical systems with an almost one-wave-cycle temporal resolution. We show that temporal focusing of a broadened pulse can be achieved for certain propagation depths and Airy-Bessel wave packets [6] of very high spatio-temporal localization can be generated by a single circular phase diffraction grating.

\section{Pulsed Bessel beam}

Under broadband illumination diffractive axicon is useful for pulsed Bessel beam generation as the resulting radial profile remains constant over entire spectrum and propagation length. However, the temporal profile of the pulsed Bessel beam is not propagation-invariant contrary to the Bessel-X pulses measured in [2,3]. Previous experiments with circular phase gratings had limited spectral range and lacked the temporal resolution to observe temporal broadening and focusing [5].

If the groove spacing of the grating is $d$, then for a given diffraction order $m$ the normally incident light acquires a radial wave vector component $k_{\perp}=m \cdot 2 \pi / d$. The resulting pulsed Bessel beam can be expressed in the form 


$$
\Psi(\rho, z, t) \propto J_{0}\left(k_{\perp} \rho\right) \int_{-\infty}^{\infty} \mathrm{d} \omega A(\omega) e^{i \omega t-i k_{z} z+i \varphi_{0}(\omega)},
$$

where $k_{\perp}^{2}=\omega^{2} / c^{2}-k_{\perp}^{2}$, and $A(\omega, z)$ is the spectral amplitude for the given diffraction order. At certain distances, the grating induced negative chirp partially compensates for the positive chirp present in the initial spectral phase $\varphi_{0}(\omega)$ due to the material dispersion. Figure 1 shows the calculated evolution of the on-axis amplitude of the electric field of the pulsed Bessel beam with various initial spectral phases. Due to the remaining cubic phase in the propagation range near the temporal focus the pulses can be described as the Airy-Bessel wave packets [7].

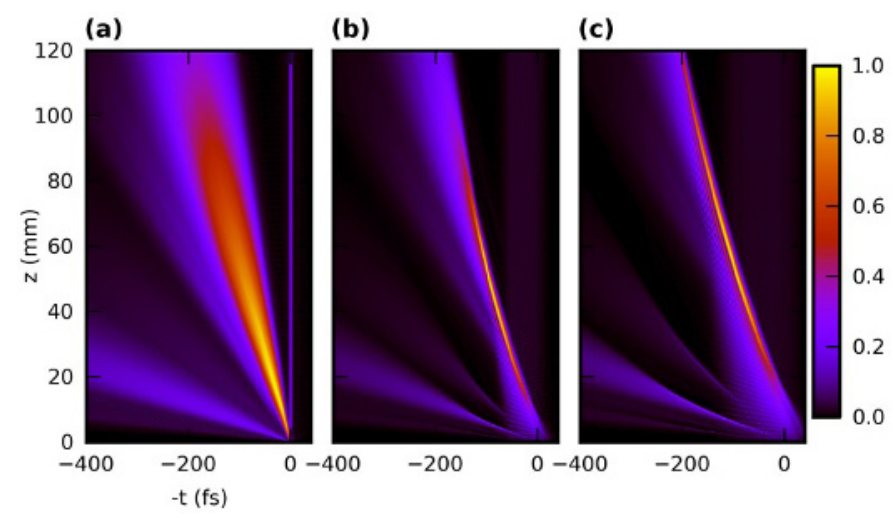

Fig. 1. The axial evolution of pulsed Bessel beam. Evaluation of Eq. (1) with various initial phases $\varphi_{0}(\omega)$ : (a) an uniform phase, i.e. no initial chirp, (b) a phase corresponding to a propagation through a fused silica sample with a thickness of $0.92 \mathrm{~mm}$, and (c) $1.57 \mathrm{~mm}$. Temporal origin corresponds to a plane wave traveling at $c$.

\section{Experimental set-up}

Our experimental set-up is presented in Fig. 2. It follows the scheme of SEA TADPOLE ultrashort pulse measurement method [1], and consists of three procedures: (i) quadratic detection of the sum of reference and unknown electric field $E_{\text {unk }}(\mathrm{r}, t)+E_{\text {ref }}(t-\tau)$, where the delay $\tau$ appears in the overlap region of the two beams tilted toward each other; (ii) temporal averaging due to the "slow" detector (a CCD camera); (iii) spectral-domain separation of the cross-correlation term from the two non-oscillating autocorrelation terms in the registered interference trace. As SEA TADPOLE had been originally developed for ultrashort pulse characterization in conjunction with FROG or another nonlinear pulse measurement method, it may have sense - for distinguishing - to call our set-up as OCEAN TADPOLE (Optical superContinuum Emission Arrangement for Noise-mediated Temporal Analysis by Dispersing a Pair of Light E-fields). In the current implementation the complex spectral response is measurable in the range of $450 \ldots 1020 \mathrm{~nm}$, resulting $\sim 3 \mathrm{fs}$ temporal and $\sim 3 \mu \mathrm{m}$ spatial resolution for pulses up to 4 ps duration.

\section{Results and conclusions}

The impulse response of a circular phase grating with a period of $20 \mu \mathrm{m}$ and various effective fused silica substrate thicknesses was measured on several distances behind the grating. Results for a $0.92 \mathrm{~mm}$ thick grating substrate are shown in Fig. 3. Initially longer pulse focuses in time at certain propagation distance $z$ and thereafter spreads out again. The experimental results and the theoretical calculations based on Eq. (1) are in good agreement.

In addition, the on-axis temporal profile of the measured pulsed responses was analyzed and FWHM duration of the pulse intensities were calculated from each measurement. The prevailing $1^{\text {st }}$ diffraction order with a transform limited duration of $3.7 \mathrm{fs}$, which initially after propagation through 
$0.92 \mathrm{~mm}$ fused silica substrate had duration of $27.8 \mathrm{fs}$, was measured to achieve the shortest duration of $5.1 \mathrm{fs}$ at $55.5 \mathrm{~mm}$ of propagation. For the $1.57 \mathrm{~mm}$ thick substrate temporal focusing from $44.8 \mathrm{fs}$ down to $6.1 \mathrm{fs}$ was observed. Especially for the latter, the quasi-Airy temporal profile could be recognized in the temporal focal region for quite long propagation extent.

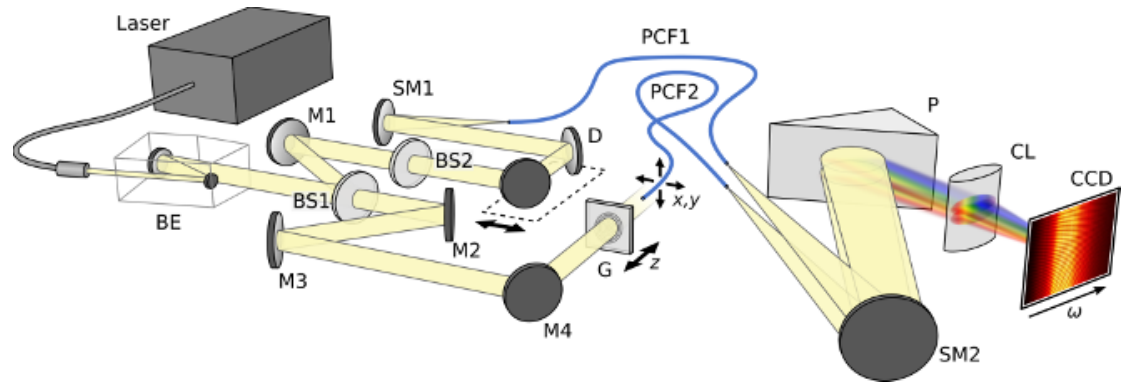

Fig. 2. Experimental set-up. The endlessly single mode photonic crystal fibres (PCF) have a few mm vertical separation on the entrance slit of the spectrometer resulting spatial interference pattern perpendicular to the frequency axis $\omega$, which carries the spectral phase and amplitude information of the field correlation function. Fibres allow effortless redesign of the set-up while keeping the spectrometer intact.

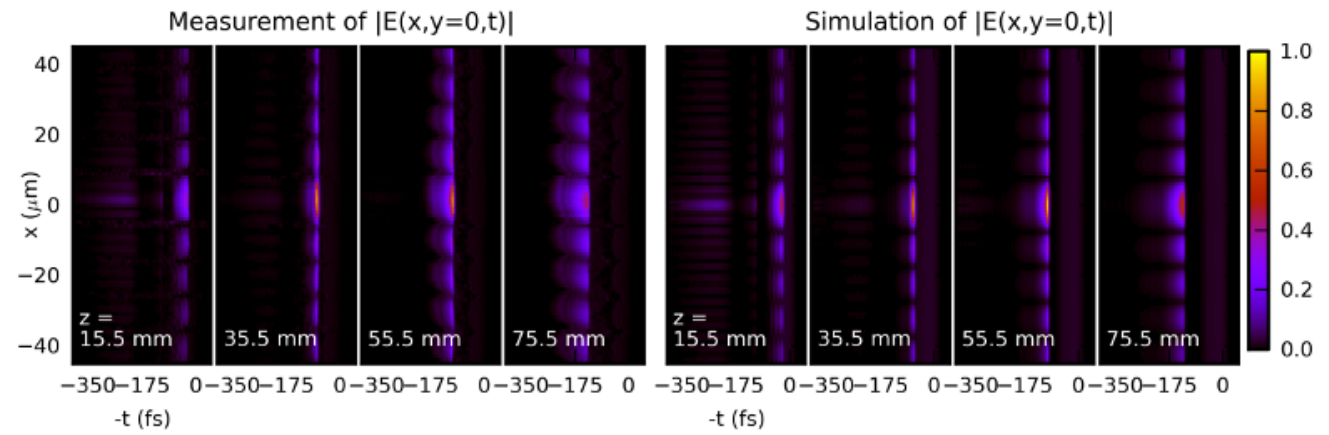

Fig. 3. Spatio-temporal evolution of the pulsed Bessel beam generated by a circularly symmetric phase grating with a substrate thickness of $0.92 \mathrm{~mm}$. Measurements performed at 4 different propagation depths. The colour shows the amplitude of the electric field. The temporal origin $t=0 \mathrm{fs}$ corresponds to a plane wave traveling at $c$.

We have studied the temporal broadening and focusing effect of ultrashort pulsed Bessel beams generated by circular gratings. We achieved 7-fold decrease in the temporal spread of ultrashort impulse response by forming the Airy-Bessel wave packet in an extremely simple set-up consisting only of one optical element-a diffractive axicon.

\section{References}

1. P. Bowlan, U. Fuchs, R. Trebino, U. D. Zeitner, Opt. Express 16, 13663 (2008)

2. H. Valtna-Lukner, P. Bowlan, M. Lõhmus, P. Piksarv, R. Trebino, P. Saari, Opt. Express 17, 14948 (2009)

3. P. Bowlan, H. Valtna-Lukner, M. Lõhmus, P. Piksarv, P. Saari, R. Trebino, Opt. Lett. 34, 2276 (2009)

4. P. Piksarv, P. Bowlan, M. Lõhmus, H. Valtna-Lukner, R. Trebino, P. Saari, J. Opt. 14, 015701 (2012)

5. M. Lõhmus, P. Bowlan, P. Piksarv, H. Valtna-Lukner, R. Trebino, P. Saari, Opt. Lett. 37, 1238 (2012)

6. A. Chong, W. H. Renninger, D. N. Christodoulides, F. W. Wise, Nature Photon. 4, 103 (2010)

7. Z. Ren, H. Jin, Y. Shi, J. Xu, W. Zhou, H. Wang, J. Opt. Soc. Am. A 29, 848 (2012) 